\title{
Competición y cooperación entre principios intrínsecos y extrínsecos de agrupamiento perceptivo en el ámbito de la visión
}

\author{
Pedro R. Montoro y Dolores Luna* \\ Departamento de Psicología Básica I, UNED, España
}

\begin{abstract}
Resumen: En el presente estudio se analizaron las interacciones entre principios de agrupamiento perceptivo intrínsecos (proximidad y semejanza) y extrínsecos (región común). En tres experimentos se presentaron los principios operando de forma aislada o en combinación bajo condiciones tanto de cooperación como de competición. Los resultados mostraron que cuando se utilizaron los principios de semejanza y región común (Experimentos 1 a y 1 b) el efecto de agrupamiento de los principios aislados fue menor que el observado en la condición de cooperación y mayor que el observado en la condición de competición. Por el contrario, cuando se utilizaron los principios de proximidad y región común (Experimento 2), el efecto de agrupamiento del principio de proximidad resultó similar al de la condición de competición. Los resultados sugieren que las interacciones entre principios podrían estar moduladas por la saliencia relativa de los principios cuando actúan de forma aislada.

Palabras clave: Organización perceptiva; agrupamiento visual; proximi-
\end{abstract} dad; semejanza; región común; saliencia.

\begin{abstract}
Title: Competition and cooperation between intrinsic and extrinsic perceptual grouping principles in vision.

Abstract: In the present study, we examined the interactions between intrinsic (proximity and similarity) and extrinsic (common region) grouping principles. In three experiments, each principle was displayed alone or conjoined with another principle both in a competitive or cooperating way. When common region and similarity principles were used (Experiments $1 \mathrm{a}$ $\& 1 \mathrm{~b})$, the results showed that the grouping strength of conjoined cooperating principles was greater than the strength of either principle alone, and the grouping strength of conjoined competing principles was lesser than that of either principle alone. In contrast, when common region and proximity principles were used (Experiment 2), the grouping effect of proximity acting alone was similar to that of competing conjoined principles. The results suggest that the interactions between grouping principles might be modulated by the relative salience of the principles acting alone.

Key words: Perceptual organization; visual grouping; proximity; similarity; common region; salience.
\end{abstract}

\section{Introducción}

Los principios de agrupamiento perceptivo permiten la percepción de la estructura del mundo visual. Aunque tradicionalmente se ha atribuido la formulación pionera de estos principios a la Teoría de la Gestalt, un estudio reciente (Vezzani, Marino y Giora, 2012), revela que ya se produjeron algunas contribuciones anteriores en el contexto de la Psicología Experimental por parte de Schuman (agrupamiento por proximidad y simetría vertical) y Müller y Rubin (agrupamiento por semejanza).

No obstante, el desarrollo más importante sobre los principios de agrupamiento perceptivo se llevó a cabo en el contexto de la Escuela de la Gestalt, a partir de las aportaciones de Max Wertheimer (1923/1938). Desde la formulación de los principios gestálticos, se ha intentado determinar la interacción entre los mismos (Koffka, 1935/1963; Köhler, 1920/1938; Wertheimer, 1923/1938) aunque el estudio cuantitativo de este problema se ha desarrollado más recientemente, a lo largo del último siglo (Wagemans et al., 2012)

Varios estudios han intentado cuantificar las interacciones entre principios de agrupamiento (Claessens y Wagemans, 2005; Hochberg y Hardy, 1960; Hochberg y Silverstein, 1956; Oyama y Miyano, 2008; Oyama, Simizu y Tozawa, 1999; Quinlan y Wilton, 1998). Los resultados de estos estudios muestran que, en general, el efecto de agrupamiento de los principios presentados aisladamente es menor que el de los principios combinados en cooperación y mayor que el efecto de agrupamiento de los principios combinados en

* Dirección para correspondencia [Correspondence address]: Dolores Luna. Departamento de Psicología Básica I. Facultad de Psicología. UNED. C/ Juan del Rosal 10. 28040, Madrid (España). E-mail: mluna@psi.uned.es competición. Estos resultados son compatibles con efectos aditivos de los principios de agrupamiento de acuerdo con los criterios establecidos por Kubovy y van den Berg (2008).

Los estudios citados anteriormente han examinado las interacciones entre principios intrínsecos (la mayoría de los principios clásicos ya formulados por la Teoría de la Gestalt) según la distinción de Palmer (1992, 1999), y en los que el agrupamiento se basa en las relaciones intrínsecas entre las propiedades de los elementos, como su forma, tamaño, posición, etc. En contraposición, en los principios que Palmer denomina extrínsecos, el agrupamiento se fundamenta en las relaciones entre las unidades estimulares discretas y otros elementos externos que las inducen a agruparse. Por ejemplo, en la dos últimas filas de la Figura 1 se puede observar cómo los elementos idénticos y con la misma distancia espacial entre ellos tienden a agruparse cuando están situados en la misma región espacial (agrupamiento por región común, Palmer, 1992) o cuando están conectados (agrupamiento por conexión, Palmer y Rock, 1994 a,b).

El reciente trabajo de Luna y Montoro (2011) ha examinado las interacciones entre principios intrínsecos (semejanza o proximidad) y el principio extrínseco de región común, utilizando el procedimiento de Quinlan y Wilton (1998), quienes estudiaron las interacciones entre el agrupamiento por proximidad y semejanza de forma o luminancia, presentando diferentes estímulos en los que los principios de agrupamiento actuaban aisladamente o combinados bajo condiciones de cooperación o competición. La tarea de los participantes consistía en evaluar, mediante una escala, el grado o intensidad de agrupamiento de un elemento central con los elementos situados a su derecha o izquierda. 


\section{口}

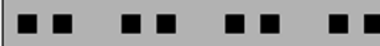
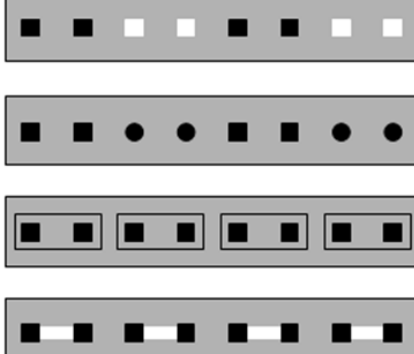

Figura 1. Ejemplos de principios de agrupamiento intrínseco y extrínseco. En la primera fila se presenta un patrón sin agrupar. En las tres siguientes se presentan ejemplos de agrupamiento intrínseco por proximidad y semejanza en color y forma, respectivamente. En las dos últimas filas se presentan ejemplos de agrupamiento extrínseco basado en región común y conexión respectivamente.

Los resultados de Luna y Montoro (2011) mostraron que, cuando los principios operaban aisladamente, el efecto de agrupamiento fue menor que cuando cooperaban y mayor que cuando competían. En general, estos resultados fueron similares a los obtenidos por Quinlan y Wilton en las interacciones entre proximidad y semejanza de color. Por otra parte, los resultados presentaron algunas diferencias con respecto a los de las interacciones entre proximidad y semejanza de forma obtenidos por dichos autores, los cuales no encontraron efecto de agrupamiento en la condición de semejanza de forma ni diferencias entre el efecto de agrupamiento en la condición de proximidad y cooperación. La falta de efecto de agrupamiento por semejanza de forma en el estudio citado de Quinlan y Wilton podría deberse a la existencia de una confusión experimental entre la forma y el tamaño (véase Figura 2 de Quinlan y Wilton, 1998, p. 420), ya que se presentaron como estímulos círculos y triángulos, y en estos últimos el tamaño en el eje vertical era mayor que el de los círculos. Además, la diferencia de resultados entre los dos estudios en relación al principio de proximidad podría deberse a la distancia entre grupos de elementos utiliza$\mathrm{da}$ (7 $\mathrm{mm}$ en Luna y Montoro y $5 \mathrm{~mm}$ en Quinlan y Wilton).

Por otra parte, las diferencias en los estímulos utilizados, igualados en saliencia visual de los principios utilizados en el estudio de Luna y Montoro (2011) y no igualados en el estudio de Quinlan y Wilton (1988), dificultan la comparación entre el patrón de resultados obtenido cuando se combinan exclusivamente principios intrínsecos entre sí y cuando se combinan principios intrínsecos con extrínsecos. La saliencia del agrupamiento puede definirse como el grado o fuerza de la impresión perceptiva con la que un grupo de dos o más elementos visuales destaca sobre el resto de elementos o grupos perceptivos (véase una revisión de Masin, 2012). La saliencia del agrupamiento puede aumentar con la cercanía de los elementos entre sí, la semejanza en forma, luminancia, color o tamaño, la pertenencia a una misma región visual o, en general, mediante cualquier otra característica visual definida por los principios de agrupamiento perceptivo.

El objetivo del presente trabajo consiste en examinar si el patrón de interacciones entre los principios intrínsecos de proximidad y semejanza y el principio extrínseco de región común, es similar o diferente al que se obtiene cuando se combinan principios intrínsecos. Para ello, (1) se utilizaron estímulos en los que se eliminó la confusión entre forma y tamaño presente en los estímulos de Quinlan y Wilton (1998) y (2) se utilizó una distancia de $5 \mathrm{~mm}$ entre subgrupos perceptivos o cohortes en el principio de proximidad, con el fin de igualar la distancia utilizada por los citados autores y así facilitar la comparación entre los dos estudios. Con este objetivo, se realizaron tres experimentos en los que principios de agrupamiento intrínseco (semejanza o proximidad) y extrínsecos (región común) se presentaron aislados o combinados bajo condiciones de: (a) cooperación, cuando dos principios de agrupamiento se combinan de forma que cooperan fortaleciendo el agrupamiento en el mismo lado del patrón y propician una organización perceptiva estable; o (b) competición, cuando los dos principios se combinan de forma que compiten entre sí por agrupar el objetivo, propiciando una organización perceptiva inestable y ambigua que fluctúa entre el agrupamiento del objetivo con los elementos situados a la derecha o a la izquierda. Ejemplos de estas condiciones se presentan en la Figura 2. Se llevaron a cabo tres experimentos diferentes. Por un lado, los experimentos $1 \mathrm{a}$ y $1 \mathrm{~b}$ examinaron las interacciones entre región común y semejanza de forma o de luminancia respectivamente. En el Experimento 2, se examinaron las interacciones entre región común y proximidad.

\section{Experimento 1a}

En este experimento se examinaron las interacciones entre el principio intrínseco de semejanza basada en la forma, mediante la presentación de círculos o cuadrados, y el principio extrínseco de región común, con el objetivo de determinar si las interacciones siguen el mismo patrón observado en investigaciones previas sobre interacciones entre principios extrínsecos o, por el contrario, presentan un patrón diferente.

\section{Método}

\section{Participantes}

Veintidós estudiantes de tercer curso de Psicología participaron en los experimentos, todos ellos presentaban visión normal o corregida. El estudio fue aprobado por el Comité Local de Ética de acuerdo con la Declaración de Helsinki.

Aparatos

Los experimentos se realizaron en sesiones colectivas en un laboratorio multimedia con una capacidad máxima de 18 
personas. Las sesiones tuvieron un aforo de entre 8 y 18 participantes. Cada sujeto realizó el experimento individualmente, observando los estímulos en un monitor en color, de17 pulgadas con una tasa de refresco de $75 \mathrm{~Hz}$ y una resolución de 1024 x 768 controlada por un ordenador ejecutando el programa E-Prime 1.2 (Psychology Software Tools, 19962002). La distancia de observación fue de aproximadamente $60 \mathrm{~cm}$. Las distancias lateral y longitudinal entre participantes fueron de aproximadamente $90 \mathrm{~cm}$. y $120 \mathrm{~cm}$., respectivamente.

\section{Estímulos}

Cada estímulo estaba formado por siete elementos organizados en una fila, el elemento central era el objetivo. Los otros seis elementos estaban organizados en dos cohortes de tres elementos cada uno, situados a la derecha o a la izquierda del objetivo. Se utilizaron como formas tanto cuadrados (10 $\mathrm{mm} \times 10 \mathrm{~mm}$ ) como círculos (10 mm de diámetro), que suponen 1.0 grado de ángulo visual a una distancia de observación de $57 \mathrm{~cm}$. La distancia entre elementos fue de 2.5 $\mathrm{mm}$. Las formas se presentaban en negro sobre un fondo blanco. Después de cada exposición estimular se presentaba, centrada una máscara que consistía en un patrón de cuadrados blancos y negros $\left(0 / 0 / 0\right.$ and $255 / 255 / 255$ RGB $^{1}$, respectivamente). El tamaño de la máscara era de $105 \mathrm{~mm}$ x 20 mm y se presentó en el centro de la pantalla, cubriendo una región ligeramente mayor que la del estímulo objetivo. El rectángulo utilizado para inducir región común tuvo un tamaño de 60 x $13 \mathrm{~mm}$ y un valor de luminancia de 192 RGB.

Se diseñaron un total de veinte estímulos diferentes, correspondientes a las distintas condiciones experimentales. Ejemplos de estos estímulos pueden consultarse en la Figura 2, panel A. Para la condición de no-agrupamiento por semejanza de forma, se diseñaron dos estímulos, uno con cuadrados $\mathrm{y}$ otro con círculos, manteniendo una forma común para los siete elementos. La condición de no-agrupamiento por región común también incluyó dos patrones similares a los anteriores pero incluyendo un rectángulo que inducía una región común para los siete elementos. La condición de región común actuando aisladamente contaba con cuatro estímulos, dos de ellos formados por cuadrados y los otros dos por círculos, y en este caso, el rectángulo incluía tan solo a cuatro de los siete elementos, ya fueran los de la derecha o de la izquierda. La condición de semejanza en forma actuando aisladamente incluía cuatro estímulos, dos de ellos en los que los que cuatro de los siete elementos eran cuadrados y otros dos en los que esos cuatro elementos eran círculos, ya fueran los de la derecha o los de la izquierda. Los cuatro estímulos correspondientes a la condición de cooperación fueron similares a los patrones de la condición de semejanz̧a en forma pero, además, in-

${ }^{1}$ En el sistema de representación RGB, los colores acromáticos obtienen valores idénticos en los tres ejes del modelo, por lo que a partir de ahora en el texto tan solo se presentará un valor asumiendo que es extensivo para los tres ejes, puesto que no se utilizó ningún color cromático en el presente estudio. cluyendo un rectángulo que contenía a los cuatro elementos con la misma forma, ya fueran círculos o cuadrados. Los cuatro estímulos correspondientes a la condición de competición también fueron similares a los patrones de la condición de semejanza en forma pero, además, incluyendo un rectángulo que encerraba en una misma región común al elemento central con los tres elementos diferentes en forma.

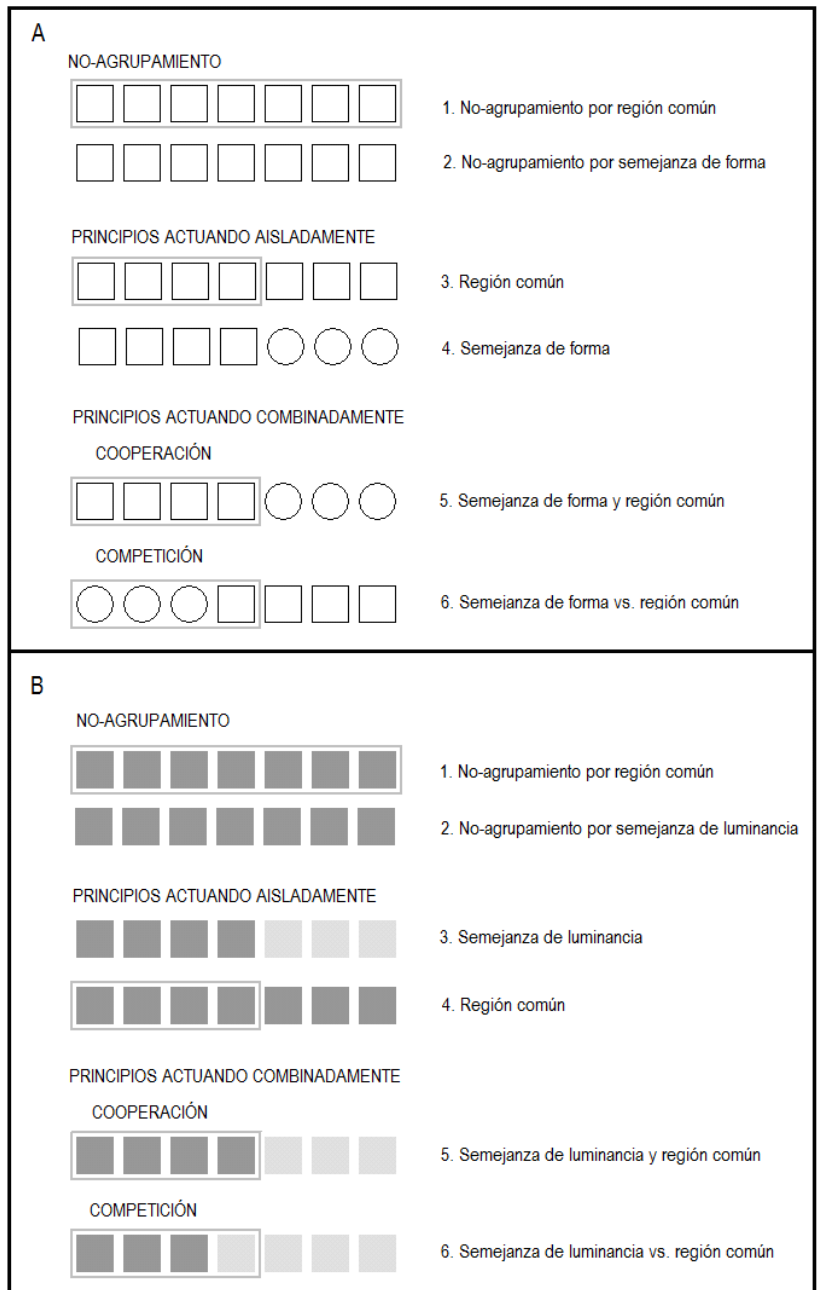

Figura 2. Ejemplos de estímulos utilizados en el Experimento 1a (Panel A) y de estímulos utilizados en el Experimento 1b (Panel B).

\section{Procedimiento y Diseño}

Al comienzo de la sesión se proporcionaron instrucciones verbales que también se presentaron escritas en la pantalla del ordenador al principio del experimento. Se instruyó a los participantes para que evaluaran mediante una escala de 9 puntos el grado o magnitud del agrupamiento del objetivo central con la cohorte de elementos situado a la derecha o a la izquierda. Se les comunicó que un valor de 5 representaba su percepción de que el objetivo no se agrupaba con ninguna de las dos cohortes. Por otro lado, valores menores de 5 implicaban que el objetivo tendía a agruparse con los ele- 
mentos de la izquierda y, por último, valores mayores que 5 que el objetivo tendía a agruparse con la cohorte de la derecha. Se explicó a los participantes que a mayor distancia numérica respecto al valor central de 5, mayor grado o fuerza de agrupamiento estaban juzgando.

Cada ensayo comenzaba con la presentación de un punto de fijación (cruz) en el centro de la pantalla; 1000 ms después, se presentaba el estímulo durante $200 \mathrm{~ms}$ y era reemplazado por la máscara que permanecía en pantalla durante 250 ms. La máscara cubría completamente la región espacial ocupada por el estímulo previo. Una vez finalizada la presentación de la máscara se presentaban nueve cuadrados que contenían dígitos del 1 al 9. Los participantes tenían que utilizar el ratón para seleccionar el cuadrado que se correspondía con su evaluación del grado de agrupamiento. Los cuadrados de respuesta se dispusieron en el centro de la pantalla, ordenados horizontalmente de izquierda a derecha, desde el número uno al nueve, respectivamente. Cada cuadrado de respuesta tenía un tamaño de $25 \mathrm{~mm}$ x $25 \mathrm{~mm}$. Una vez finalizada esta secuencia comenzaba un nuevo ensayo. Los estímulos se presentaron enmascarados y con tiempo breves de exposición para inducir a los participantes a responder conforme a su impresión inmediata de los estímulos.

Cada bloque de ensayos constaba de una presentación aleatoria de todos los estímulos correspondientes a cada experimento. Se dispensaron cinco bloques experimentales y un bloque de 24 ensayos de práctica. Cada bloque experimental constaba de 48 ensayos. En cada bloque, los estímulos sin agrupamiento se repitieron cuatro veces cada uno de ellos y se presentó la misma proporción de estímulos a la derecha que de estimulos a la izquierda.

En cada experimento se presentaron cuatro condiciones experimentales correspondientes a los estímulos descritos: (1) no-agrupamiento, (2) principios operando aisladamente y principios combinados en (3) cooperación o (4) competición. Ejemplos de estas condiciones pueden observarse en las Figuras 2 y 5.

\section{Resultados}

En todos los experimentos, las puntuaciones directas fueron transformadas siguiendo el procedimiento descrito por Quinlan y Wilton (1998). Para ello, se definió una cohorte dominante que contenía el objetivo central en cada estímulo. En el caso de los principios que actuaban aisladamente y en la condición de cooperación, la cohorte dominante fue determinada por el principio o principios de agrupamiento implicados. Por ejemplo, en el Experimento 1a, la cohorte dominante en los estímulos correspondientes al principio de región común es la que está dentro del mismo rectángulo que el objetivo (Estímulo 3 en el Panel A de la
Figura 2), mientras que en los estímulos correspondientes a forma, se definió como aquella en que los elementos tienen la misma forma que el objetivo (Estímulo 4 en el Panel A de la Figura 2). En los estímulos correspondientes a condiciones de cooperación, la cohorte dominante se definió por los dos principios combinados, por ejemplo, región común y semejanza en forma en el Experimento 1a (Estímulo 5 del Panel A de la Figura 2). En cambio, en el caso de la condición de competición, la cohorte dominante fue arbitrariamente asignada a la cohorte agrupada mediante región común.

Las puntuaciones directas fueron convertidas a una nueva escala de -4 a +4 puntos, que reflejaba el grado o magnitud del agrupamiento del objetivo central con la cohorte dominante $(+1 \mathrm{a}+4)$ o con la cohorte alternativa (-4 a -1$)$. Los análisis presentados a continuación fueron realizados con estas puntuaciones transformadas.

En los tres experimentos los datos correspondientes a las condiciones de agrupamiento se analizaron de la forma descrita a continuación. En primer lugar, la valoración media observada se comparó con un valor esperado de 0 por medio del estadístico $t$ para una muestra. En segundo lugar, se realizó un ANOVA para determinar si el efecto de agrupamiento presentaba diferencias entre las condiciones en que el agrupamiento resultó significativo en el análisis anterior. Finalmente, para comparar el tamaño del efecto de agrupamiento en las seis condiciones se realizaron comparaciones post-hoc aplicando correcciones de Bonferroni. A continuación se presentan los resultados de los análisis para cada experimento.

Los principales resultados se presentan en la Figura 3. El efecto de agrupamiento resultó significativo en las condiciones de agrupamiento basado en la semejanza de forma, $t(21)$ $=17.60, p<.001$; región común, $t(21)=16.66, p<.001 \mathrm{y}$ cooperación, $t(21)=25.00, p<.001 ; \mathrm{y}$ no significativo en la condición de competición $t(21)=1.5, p>.10$. Con el fin de examinar si la magnitud del efecto de agrupamiento variaba entre las condiciones, se realizó un ANOVA sobre las condiciones en las que se observó un efecto significativo del agrupamiento, excluyéndose la condición de competición. Este análisis reveló un efecto principal significativo de las condiciones de agrupamiento, $F(2,42)=5.82, p=.006$. Finalmente las comparaciones post hoc revelaron los siguientes resultados: el efecto de agrupamiento fue similar en las condiciones en que los principios de agrupamiento basado en la semejanza de forma y región común operaban aisladamente $t(21)=-.19, p=1$. El efecto de agrupamiento en la condición de cooperación fue mayor que el de la condición de región común $(t(21)=-.4 .03, p=.002)$ o forma $t(21)=$ $-2.78, p=.034$. 


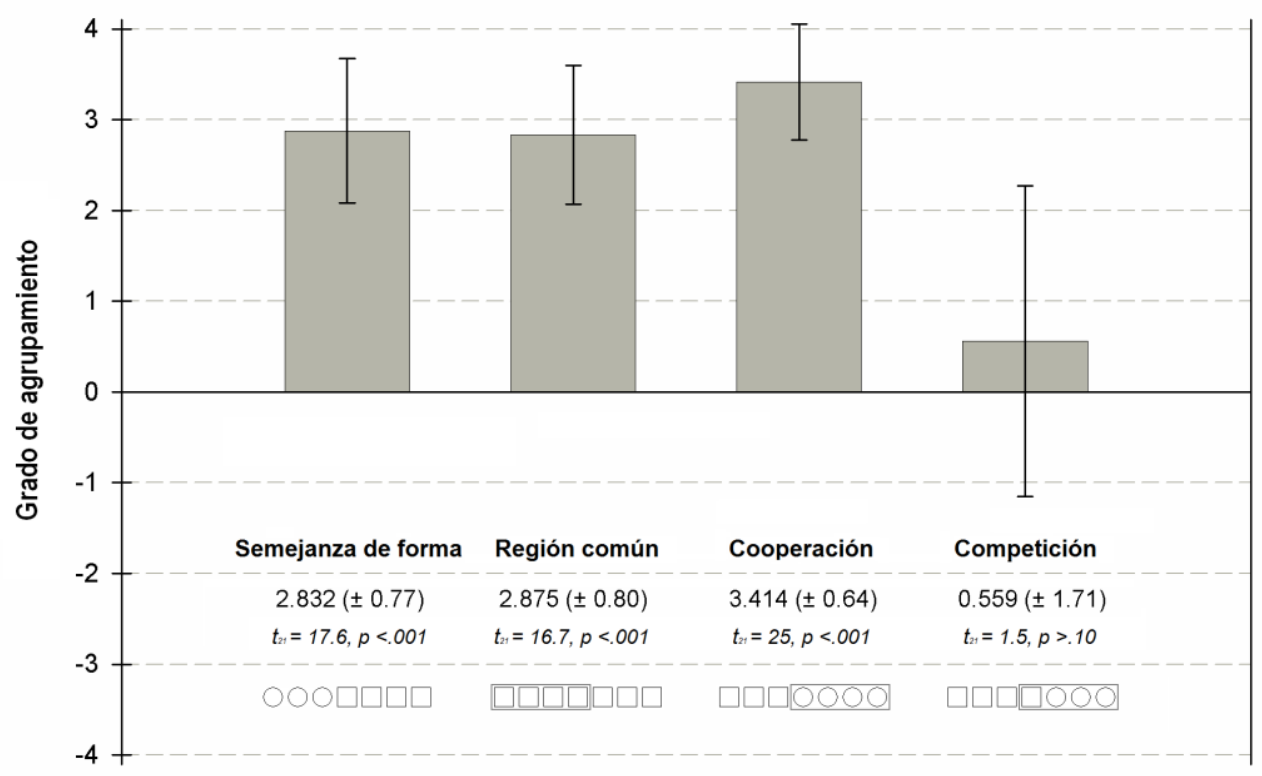

Figura 3. Medias de las puntuaciones transformadas del grado de agrupamiento del Experimento 1a. Las barras representan \pm 1 desviaciones típicas.

\section{Experimento 1b}

En el presente experimento se examinaron las interacciones entre el principio intrínseco de semejanza basada en luminancia y el principio extrínseco de región común.

\section{Método}

\section{Participantes}

Veintidós estudiantes de tercer curso de Psicología participaron en los experimentos, todos ellos presentaban visión normal o corregida. El estudio fue aprobado por el Comité Local de Ética de acuerdo con la Declaración de Helsinki.

\section{Aparatos}

Los aparatos fueron los mismos que los utilizados en el Experimento 1a.

\section{Estímulos}

Se presentaron un total de veinte estímulos diferentes, correspondientes a las distintas condiciones experimentales. Ejemplos de estos estímulos pueden consultarse en la Figura 2, panel B. Para la condición de no-agrupamiento por semejanza de luminancia, se diseñaron dos estímulos, uno formado por siete elementos con un valor de luminancia de 220 RGB y otro con elementos con una luminancia de 148 RGB. La condición de no-agrupamiento por región común también incluyó dos patrones similares a los anteriores pero incluyendo un rectángulo que inducía una región común para los siete elementos. La condición de región común actuando aisladamente contaba con cuatro estímulos, dos de ellos formados por elementos con un valor de luminancia de 220 RGB y los otros dos con una luminancia de 148 RGB, y en esta condición, el rectángulo incluía tan solo a cuatro de los siete elementos, ya fueran los de la derecha o de la izquierda. La condición de semejanza de luminancia actuando aisladamente incluía cuatro estímulos, dos de ellos en los que los que cuatro de los siete elementos tenían una luminancia de 220 RGB y otros dos en los que esos cuatro elementos tenía un luminancia de 148 RGB, ya fueran los de la derecha o los de la izquierda. Los cuatro estímulos correspondientes a la condición de cooperación fueron similares a los patrones de la condición de semejanza de luminancia pero, además, incluyendo un rectángulo que contenía a los cuatro elementos con el mismo valor de luminancia, ya fuera 220 RGB o 148 RGB. Los cuatro estímulos correspondientes a la condición de competición también fueron similares a los patrones de la condición semejanza de luminancia pero, además, incluyendo un rectángulo que encerraba en una misma región común al elemento central con los tres elementos diferentes en su valor de luminancia. El patrón de máscara estuvo formado por una matriz de 29 × 8 de cuadrados con tres valores de luminancia distribuidos alternativamente (142, 184 and 220 RGB) y con una proporción semejante de los tres. El rectángulo utilizado para inducir región común fue idéntico al utilizado en el Experimento 1a. 


\section{Procedimiento}

El procedimiento fue similar al descrito en el Experimento 1a con la excepción de que los estímulos fueron diferentes.

\section{Resultados}

Los resultados fueron semejantes a los encontrados en el Experimento 1a y se presentan en la Figura 4. El efecto de agrupamiento resultó significativo en las condiciones de: agrupamiento basado en luminancia, $t(21)=25.02, p<.001$; región común, $t(21)=15.72, p<.001$ y cooperación, $t(21)$ $=51.43, p<.001$. El efecto no resultó significativo en la condición de competición, $t(21)=-1.425, p=.17$. El ANOVA realizado sobre las condiciones en las que el efecto de agrupamiento resultó significativo reveló un efecto principal significativo de las condiciones de agrupamiento $F$ $(2,42)=18.51, p<.001$. Las comparaciones post hoc revelaron los siguientes resultados: el efecto de agrupamiento fue similar en las condiciones en que los principios de agrupamiento basado en la semejanza en luminancia y región común operaban aisladamente, $t(21)=-2.18, p=.12$. El efecto de agrupamiento en la condición de cooperación fue mayor que el de la condición de región común $(t(21)=-5.85, p=$ $.001)$; o semejanza de luminancia $(t(21)=-5.78, p=.001$.

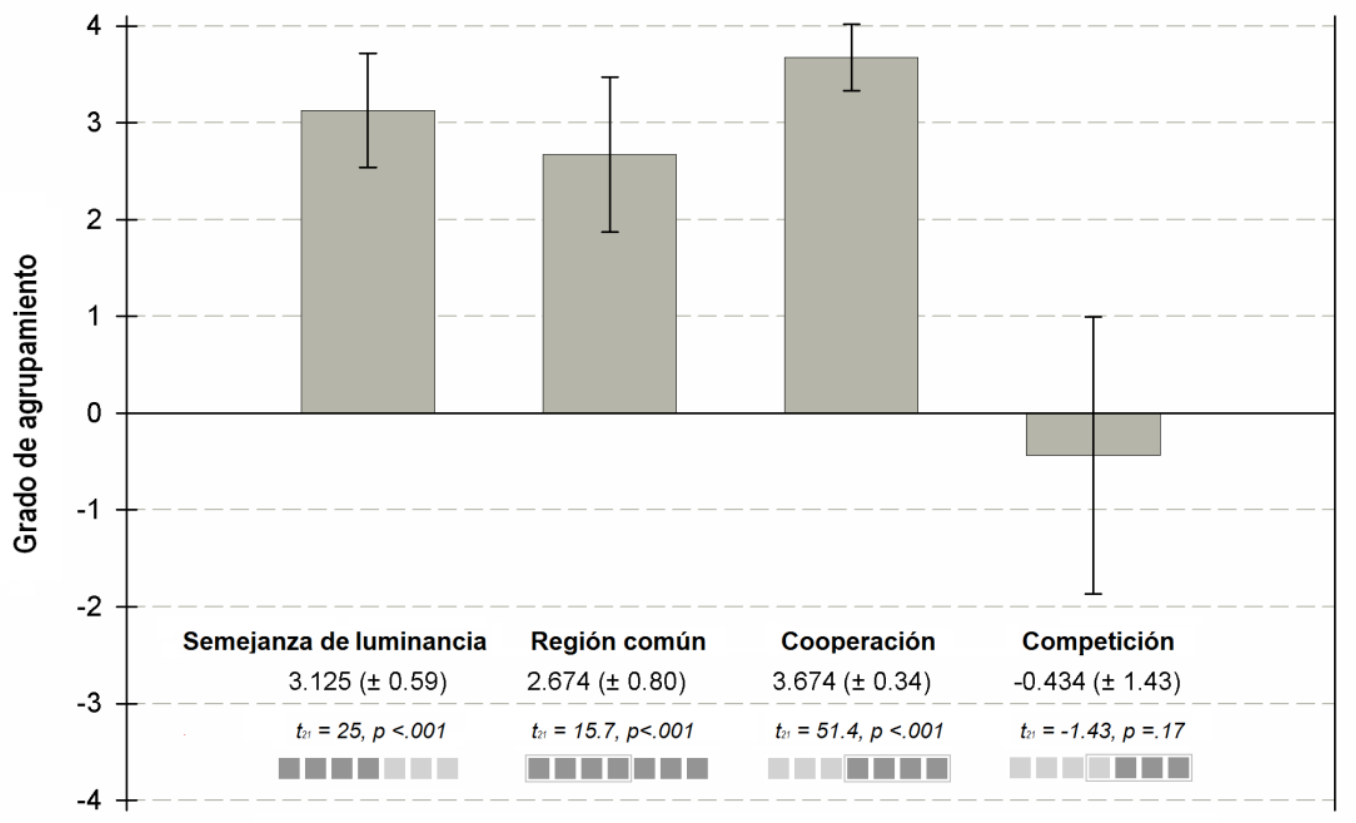

Figura 4. Medias de las puntuaciones transformadas del grado de agrupamiento del Experimento $1 \mathrm{~b}$.

$$
\text { Las barras representan } \pm 1 \text { desviaciones típicas. }
$$

En los experimentos 1a y $1 \mathrm{~b}$ no hubo efecto de agrupamiento en la condición de competición. Sin embargo, este resultado no se debió a que los participantes puntuaran 0 en esta condición, sino a que eligieron uno u otro principio para agrupar el objetivo. En el Experimento 1a, ocho participantes tendieron a agrupar por semejanza en forma y catorce por región común. En el experimento $1 \mathrm{~b}$, diez participantes tendieron a agrupar por región común y once por semejanza en luminancia y únicamente un sujeto puntuó cero en la condición de competición. Por lo tanto, la obtención de un efecto nulo de agrupamiento en la condición de competición deriva de un fenómeno grupal, consistente en una amplia dispersión de la respuesta entre los observadores, como refleja el elevado valor de desviación típica en dicha condición $(D T=1.71$; véase Fig. 4

\section{Experimento 2}

En el experimento 2 se examinaron las interacciones entre el principio intrínseco de proximidad y el principio extrínseco de región común. En este caso, y a diferencia de los dos experimentos anteriores, el agrupamiento en ambos principios está basado en relaciones espaciales entre los elementos: distancia entre elementos en el caso de proximidad y relación de los elementos con el elemento externo (rectángulo en el que están incluidos) que los induce a agruparse por región común. 


\section{Método}

\section{Participantes}

Veintidós estudiantes de tercer curso de Psicología participaron en los experimentos, todos ellos presentaban visión normal o corregida. El estudio fue aprobado por el Comité Local de Ética de acuerdo con la Declaración de Helsinki.

\section{Aparatos}

Los aparatos fueron los mismos que los utilizados en los Experimentos 1a y $1 \mathrm{~b}$.

\section{Estímulos}

Se presentaron un total de diez estímulos diferentes, correspondientes a las distintas condiciones experimentales, todos ellos formados exclusivamente por cuadrados. Ejemplos de estos estímulos pueden consultarse en la Figura 5. Para las condiciones de no-agrupamiento por proximidad y por región común se utilizaron dos estímulos, uno para cada condición, idénticos a esas mismas condiciones del Experimento1a, pero solo conteniendo cuadrados, con una distancia homogénea entre elementos de $2.5 \mathrm{~mm}$. Del mismo modo, la condición de región común actuando aisladamente contaba con dos estímulos idénticos a los de la misma condición del Experimento 1a, pero solo formada por cuadrados. La condición de proximidad actuando aisladamente incluía dos estímulos, de forma que en ambos un grupo de cuatro elementos se desplazó $5 \mathrm{~mm}$. hacia la derecha o hacia la izquierda de los tres elementos restantes. Los dos estímulos correspondientes a la condición de cooperación fueron similares a los patrones de la condición de proximidad actuando aisladamente pero, además, incluyendo un rectángulo que contenía a los cuatro elementos desplazados. Los dos estímulos correspondientes a la condición de competición también fueron similares a los patrones de la condición de proximidad actuando aisladamente pero, además, incluyendo un rectángulo que encerraba en una misma región común al elemento central con los tres elementos más alejados del mismo. Las características del patrón de máscara y del rectángulo utilizado para inducir una región común fueron idénticas a las del Experimento 1a. El rectángulo utilizado para inducir región común fue idéntico al utilizado en los Experimentos 1a y 1b.

\section{Procedimiento}

El procedimiento fue similar al descrito en el Experimento 1a con la excepción de que los estímulos fueron diferentes y que cada bloque experimental en el Experimento 2 constaba de 24 ensayos.

\section{NO-AGRUPAMIENTO}

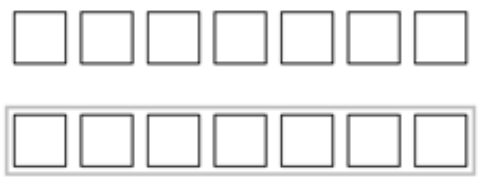

PRINCIPIOS ACTUANDO AISLADAMENTE

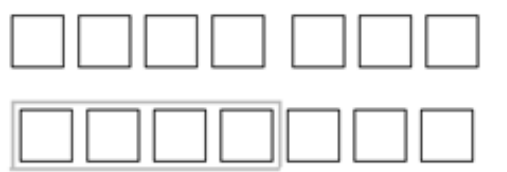

PRINCIPIOS ACTUANDO COMBINADAMENTE COOPERACIÓN

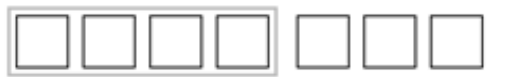

COMPETICIÓN

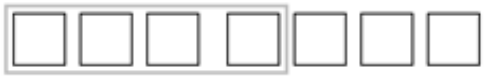

1. No-agrupamiento por proximidad

2. No-agrupamiento por región común

3. Proximidad

4. Región

5. Proximidad y región común

6. Proximidad vs, región común

Figura 5. Ejemplos de estímulos presentados en el Experimento 2. Los estímulos 1 y 2 son ejemplos de condiciones de no-agrupamiento, los estímulos 3 y 4 son ejemplos de principios de agrupamiento operando aisladamente, el estímulo 5 es un ejemplo de la condición de cooperación y el 6 de la condición de competición. 


\section{Resultados}

En la Figura 6, se presentan los resultados más importantes observados en este experimento. Los resultados fueron diferentes a los encontrados en los Experimentos 1a y 1b. En este experimento el efecto de agrupamiento fue significativo en todas las condiciones: proximidad, $t(21)=14.04$, $p<.001$ ); región común $t(21)=25.07, p<.001$; cooperación, $t(21)=28.14, p<.001$; y competición $t(21)=6.36, p<.001$. El ANOVA mostró un efecto significativo principal de las condiciones de agrupamiento, $F(2,42)=22.917, p<.001$. Las comparaciones post hoc pusieron de manifiesto los siguientes resultados. El efecto de agrupamiento fue mayor en la condición de cooperación que en región común, $t$ (21)
$=9.72, p<.001$, también fue mayor que en proximidad, $t$ $(21)=11.76, p=<.003$, y que competición, $t(21)=5.15, p$ $<.001$. Por otra parte, el efecto de agrupamiento fue mayor en la condición de región común que en la de proximidad, $t$ (21) $=11.76, p=.003$, y el efecto de agrupamiento de la condición de proximidad no se diferenció del de competición, $t(21)=-0.06, p=1$.

En contraste con los experimentos 1a y 1b, los resultados en este experimento mostraron que la condición de proximidad y competición, no difirieron en su efecto de agrupamiento. Además de este resultado inesperado, prácticamente todos los sujetos (21 participantes) agruparon por región común en la condición de competición y solo uno de ellos por proximidad.

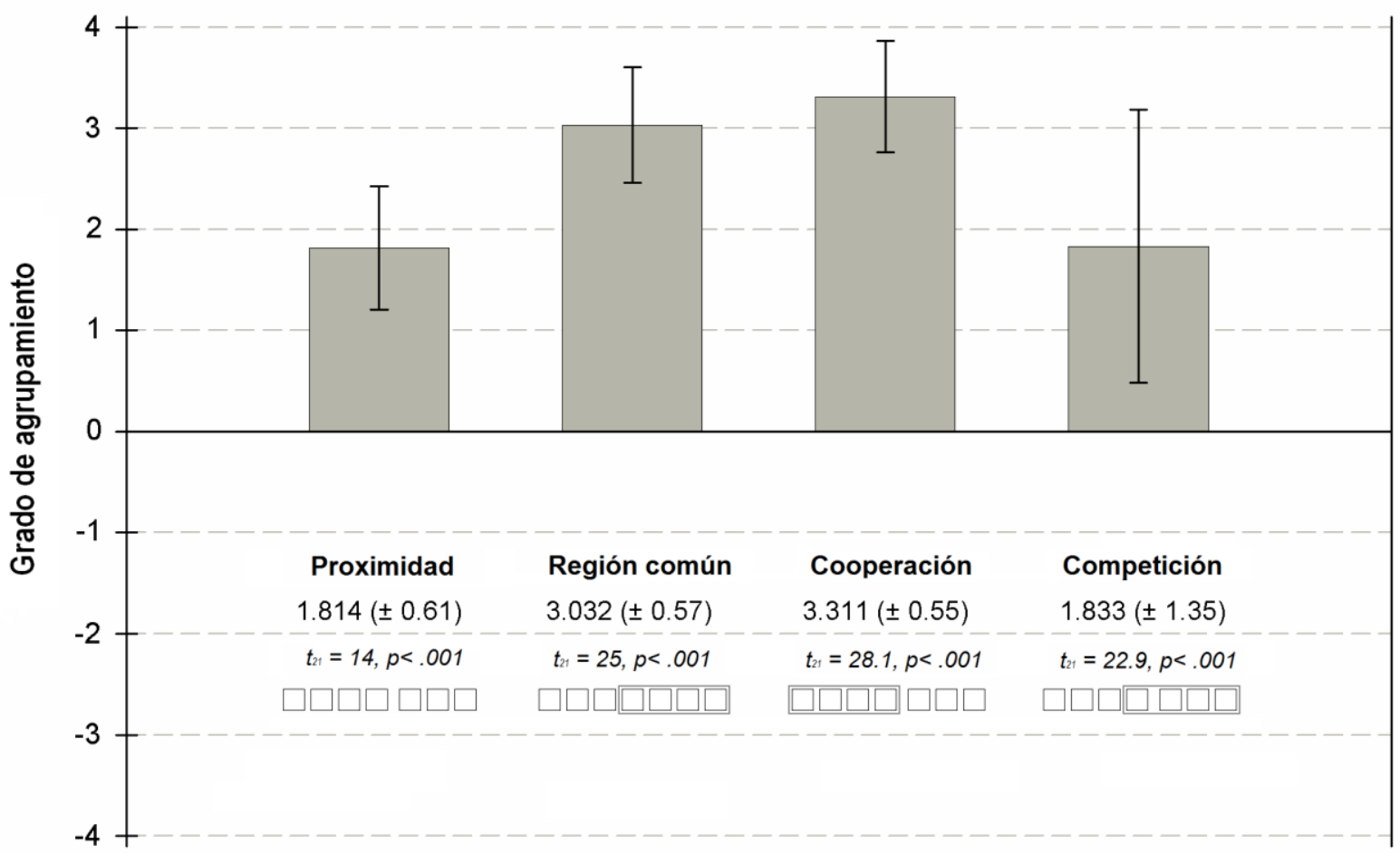

Figure 6. Medias de las puntuaciones transformadas del grado de agrupamiento del Experimento 2. Las barras representan \pm 1 desviaciones típicas.

Análisis conjunto de los datos de las condiciones de noagrupamientos

Finalmente Los datos de las condiciones de noagrupamiento de los tres experimentos se analizaron conjuntamente con el fin de examinar si se había producido algún sesgo sistemático en las respuestas. Las puntuaciones transformadas de esta condición se presentan en la Tabla 1. Una puntuación de 0 indicaba que no había sesgo, una puntuación positiva significaba sesgo hacia la cohorte izquierda y una puntuación negativa sesgo hacia la cohorte izquierda.
Los datos de las condiciones de no-agrupamiento se compararon con un valor de 0 por medio de una prueba $t$. Ninguna de estas pruebas resultó significativa (todas las $p>.10$ ), por lo que no se observó un sesgo sistemático en estas condiciones.

Los datos de los tres experimentos se analizaron por medio de un ANOVA mixto 2 × 3 con un factor intrasujetos de no-agrupamiento (no-agrupamiento-intrínseco vs. noagrupamiento extrínseco) y los tres experimentos considerados como factor entre sujetos (Experimentos $1 a, 1 b, y$ 2). El análisis reveló la ausencia de efecto significativo de las condicio- 
nes de no-agrupamiento, $F(1,63)=1.28, p=.26$, así como de la interacción entre condiciones de no-agrupamiento y experimento, $F(2,63)=2.11, p=0.12$.

Tabla 1. Medias y desviaciones típicas $(D T)$ de las puntuaciones transformadas del grado de agrupamiento ante estímulos no-agrupados en cada experimento $(\mathrm{RC}=$ región común)

\begin{tabular}{lllll}
\hline & \multicolumn{4}{l}{ Estímulos no-agrupados } \\
\cline { 2 - 5 } Experimento & \multicolumn{3}{l}{ Intrínsecos } & \multicolumn{3}{l}{ Extrínseco } \\
\cline { 2 - 5 } M & $D T$ & $M$ & $D T$ \\
\hline 1a. Semejanza de forma y RC & 0.16 & 0.04 & 0.33 & 0.32 \\
1b. Semejanza de luminancia y RC & 0.01 & 0.09 & -0.05 & 0.06 \\
2. Proximidad y RC & 0.06 & 0.18 & 0.01 & 0.10 \\
\hline
\end{tabular}

\section{Discusión general}

El objetivo del presente estudio consistió en examinar las interacciones entre principios de agrupamiento intrínseco (proximidad y semejanza de forma y luminancia) y el principio extrínseco de región común.

El patrón de resultados fue diferente en función del principio de agrupamiento que interactuaba con región común. En el caso de la semejanza, los resultados de los Experimentos 1a (combinaciones de semejanza de forma y región común) y $1 \mathrm{~b}$ (combinaciones de semejanza de luminancia y región común) revelaron efectos de agrupamiento en los principios de región común, semejanza de forma y semejanza de luminancia, cuando estos principios operaban aisladamente. Además, el efecto de agrupamiento fue similar en todos ellos, lo que indica que los dos principios que interactuaban en cada experimento presentaban la misma saliencia. También se observó un efecto de agrupamiento en las condiciones de cooperación entre principios, y este efecto resultó mayor que el obtenido cuando los principios actuaban aisladamente. Por el contrario, no se observó efecto de agrupamiento en las condiciones de competición entre principios. En el segundo caso, cuando se combinaron el principio de región común y el de proximidad en el Experimento 2, también se observó efecto de agrupamiento en los principios de región común y proximidad cuando operaban aisladamente, pero, en contraste con los experimentos $1 \mathrm{a}$ y $1 \mathrm{~b}$, el efecto de agrupamiento fue mayor en la condición de región común que en la de proximidad. El efecto de agrupamiento se observó, de igual manera, en las condiciones de cooperación y sorprendentemente también en la de competición. Finalmente el efecto de agrupamiento en la condición de cooperación resultó mayor que en el resto de condiciones y el efecto de agrupamiento en la condición de proximidad no se diferenció del observado en la condición de competición.

A diferencia de los resultados encontrados por Quinlan y Wilton (1998), en los que no se obtuvo un efecto de agrupamiento cuando el principio de semejanza en forma operaba aisladamente, en el presente estudio se observó un efecto de agrupamiento en esta condición. Este resultado se debe al hecho de que en nuestros estímulos se eliminó la confusión entre forma y tamaño presente en los estímulos utilizados por los autores citados.

Un resultado importante e inesperado en el presente estudio, que también refleja diferencias entre las combinaciones de principios intrínsecos de semejanza o proximidad con el principio extrínseco de región común, está relacionado con la predominancia de los principios en la condición de competición en función de la saliencia de los principios cuando operan aisladamente. Cuando estos principios tienen la misma saliencia, como se observa en los Experimentos 1a y $1 \mathrm{~b}$, al interactuar en la condición de competición, la organización perceptiva del patrón estimular resulta ambigua y fluctúa entre el agrupamiento del objetivo central con los elementos situados a la derecha o a la izquierda. Como consecuencia, algunos participantes tienden a agrupar de manera consistente el objetivo central basándose en el principio de región común mientras que el resto de participantes tiende a agruparlo de acuerdo con el principio de semejanza. En este caso no se da una predominancia clara de principios extrínsecos o intrínsecos en la condición de competición y el resultado promediado es un empate entre principios que propicia una ausencia de efecto de agrupamiento en la condición de competición.

Sin embargo cuando los principios intrínsecos y extrínsecos presentan importantes diferencias en saliencia, como sucede en el Experimento 2 con el principio de región común, que muestra mayor saliencia que el principio de proximidad, el principio más saliente gana la competición y el objetivo central se agrupa con los elementos más distantes situados en la región común. En este caso, prácticamente todos los participantes tienden a agrupar por región común y este principio predomina sobre el de proximidad cuando se combinan en la condición de competición.

Por lo tanto, los resultados del presente estudio sugieren que la saliencia de agrupamiento perceptivo en condiciones de competición entre principios podría depender de la saliencia relativa de los principios considerados aisladamente. De esta forma, los valores de magnitud del agrupamiento observados para cada principio actuando de manera aislada permitirían predecir el efecto del agrupamiento en la condición de competición, en línea con un modelo de efectos aditivos de los principios de agrupamiento (Kubovy y van den Berg, 2008).

Los resultados del presente trabajo son congruentes con el estudio previo de Luna y Montoro (2011) en relación al papel de la saliencia individual de los principios de agrupamiento en la interacción competitiva entre principios. Sin embargo, en ninguno de estos dos trabajos se manipula experimentalmente la saliencia de los principios de agrupamiento implicados, puesto que Luna y Montoro (2011) bloquean la saliencia de los mismos mientras que el presente trabajo mide la saliencia sin manipularla explícitamente. En vista del importante papel de este factor, son necesarios nuevos estudios en los que la saliencia relativa de principios intrínsecos y extrínsecos sea manipulada como un factor experimental (Montoro y Luna, en revisión). 


\section{Referencias}

Claessens, P. M. y Wagemans, J. (2005). Perceptual grouping in Gabor lattices: Proximity and alignment. Perception and Psychophysics, 67, 1446-1459.

Hochberg, J. y Hardy, D. (1960). Brightness and proximity factors in grouping" Perceptual \& Motor Skills, 10, 22.

Hochberg, J. y Silverstein, A. (1956). A quantitative index of stimulus-similarity: Proximity vs. differences in brightness. American Journal of Psychology, 69(3), 456-458.

Koffka, K. (1935/1963). Principles of Gestalt Psychology. New York: Harcourt, Brace \& World (Trabajo original publicado en 1935).

Köhler, W. (1920/1938). Physical gestalten at rest and in steady state: A naturalphilosophical investigation. En W. D. Ellis (Ed.), A Source Book of Gestalt Psychology, London: Routledge (Reimpresión en Die Physischen Gestalten in Rube und im stationären Zustand. Eine nature-philosophische Untersuchung, por W Kölher 1920, Braunsschweig Germany: Friedr, Vieweg und Sohn).

Kubovy, M. y van den Berg, M. (2008). The whole is equal to the sum of its parts: a probabilistic model of grouping by proximity and similarity in regular patterns. Psychological Review, 115, 131-154.

Luna, D. y Montoro, P. R. (2011). Interactions between intrinsic principles of similarity and proximity and extrinsic principle of common region in visual perception. Perception, 40, 1467-1477.

Masin, S. C. (2012). Grouping by achromatic color and surface segregation. Psychological Research, 76, 1-7.

Montoro, P. R. y Luna, D. (2014). Does the relative strength of visual grouping principles modulate cooperation and competition between them? A study of grouping by proximity and common region. Spanish Journal of Psychology, en revisión.
Oyama, T. y Mivano, H. (2008). Quantification of Gestalt laws and proposal of a perceptual state-space model. Gestalt Theory, 30, 29-38.

Oyama, T., Simizu, M. y Tozawa, J. (1999). Effects of similarity on apparent motion and perceptual grouping. Perception, 28, 739-748.

Palmer, S. E. (1992). Common region: A new principle of perceptual grouping. Cognitive Psychology, 24, 433-447.

Palmer, S. E. (1999) Vision Science. Photons to Phenomenology. Cambridge, Massachusetts: The MIT Press.

Palmer, S. E. y Rock, I. (1994a). On the nature and order of organizational processing: A reply to Peterson. Psychonomic Bulletin and Review, 1, 515-519.

Palmer, S. E. y Rock, I. (1994b). Rethinking perceptual organization: The role of uniform connectedness. Psychonomic Bulletin and Review, 1, 29-55.

Quinlan, P. T. y Wilton, R. L. (1998). Grouping by proximity or similarity? Competition between the Gestalt principles in vision. Perception, 27, 417-430.

Vezzani, S., Marino, B. F. M. y Giora, E. (2012). An early history of the Gestalt factors of organisation. Perception, 41, 148-167.

Wagemans, J., Elder, J. H., Kubovy, M., Palmer, S. E., Peterson, M. A., Singh, M. y von der Heydt, R. (2012). A century of Gestalt psychology in visual perception: I. Perceptual grouping and figure-ground organization. Psychological Bulletin, 138, 1172-1217.

Wertheimer, M. (1923/1938). Investigations of the principles of gestalt II. En W. D. Ellis (Ed.), A source book of Gestalt psychology (pp. 71-98) New York: Harcourt Brace

(Artículo recibido: 21-1-2013; revisión recibida: 4-7-2013; aceptado: 30-10-2013) 